\title{
Spinal Extradural Arachnoid Cyst
}

\author{
Joon Bum Woo, MD, Dong Wuk Son, MD, PhD, Kyung Taek Kang, MD, Jun Seok Lee, MD, \\ Geun Seong Song, MD, PhD, Soon Ki Sung, MD, PhD, and Sang Weon Lee, MD, PhD \\ Department of Neurosurgery, Pusan National University Yangsan Hospital, Yangsan, Korea
}

\begin{abstract}
A spinal extradural arachnoid cyst (SEAC) results from a rare small defect of the dura matter that leads to cerebrospinal fluid accumulation and communication defects between the cyst and the subarachnoid space. There is consensus for the treatment of the dural defect, but not for the treatment of the cyst. Some advocate a total resection of the cysts and repair of the communication site to prevent the recurrence of a SEAC, while others recommended more conservative therapy. Here we report the outcomes of selective laminectomy and closure of the dural defect for a 72-year-old and a 33-year-old woman. Magnetic resonance imaging of these patients showed an extradural cyst from T12 to L4 and an arachnoid cyst at the posterior epidural space of T12 to L2. For both patients, we surgically fenestrated the cyst and repaired the dural defect using a partial hemi-laminectomy. The patient's symptoms dramatically subsided, and follow-up radiological images show a complete disappearance of the cyst in both patients. Our results suggest that fenestration of the cyst can be a safe and effective approach in treating SEACs compared to a classical complete resection of the cyst wall with multilevel laminectomy.
\end{abstract}

(Korean J Neurotrauma 2016;12(2):185-190)

KEY WORDS: Arachnoid cyst · Fenestration, Labyrinth · Cerebrospinal fluid.

\section{Introduction}

A spinal extradural arachnoid cyst (SEAC), which is mostly found at the thoracic spine, is a rare disease, accounting for approximately $1 \%$ to $3 \%$ of spinal tumors. ${ }^{1-9)}$ SEAC patients are generally men in their 20 's, ${ }^{3)} 30$ 's, or 50's. ${ }^{6}$ SEACs are most commonly found in the middle or lower thoracic spine, and less frequently found in the lumbar region, although they can be detected at any lesion of the spine., ${ }^{1,5,6,8)}$

A SEAC results from a small defect in the dura matter that induces cerebrospinal fluid (CSF) accumulation and arachnoid membrane herniation. ${ }^{1,2,5,6,9)}$ The cause of this hernia-

Received: August 29, 2016 / Revised: October 13, 2016 Accepted: October 20, 2016

Address for correspondence: Dong Wuk Son Department of Neurosurgery, Pusan National University Yangsan Hospital, 20 Geumo-ro, Mulgeum-eup, Yangsan 50612, Korea

Tel: +82-55-360-2126, Fax: +82-55-360-2156

E-mail: md6576@naver.com

$@$ This is an Open Access article distributed under the terms of Creative Attributions Non-Commercial License (http://creativecommons. org/licenses/by-nc/3.0/) which permits unrestricted noncommercial use, distribution, and reproduction in any medium, provided the original work is properly cited. tion, which is still under debate, may be either congenital or acquired. ${ }^{1,39}$ The neurological symptoms of a SEAC, including myelopathy, can be triggered by the mass effect of cord or root compression. ${ }^{1-7)}$ A SEAC can be enlarged during exercise or other actions because there is small defect between the cysts and subarachnoid space that allows increased cranial pressure to stimulate the flow of CSF from the intradural space to the cyst. ${ }^{1,3)}$ A SEAC is a meningeal cyst that can be classified into three major categories. Type I cysts are composed of extradural arachnoid cysts (EAC) without nerve root fibers, Type II cysts are composed of EAC with nerve root fibers, and Type III cysts are composed of intradural meningeal cyst. Type 1 meningeal cysts can be subcategorized as EAC (Type Ia) and sacral meningoceles (Type Ib). ${ }^{2,5,6)}$ A SEAC can be caused by herniation of the dura matter, which can be the result of a congenital anomaly, trauma, infection, or inflammation. However, the causes of such herniation remain unclear. ${ }^{1,5,8)}$

To treat a SEAC, diverse surgical techniques have been introduced, although there is no consensus regarding the most reasonable treatment. ${ }^{1,2,5)}$ Most investigators agree that the dural defect must be repaired, but total removal of a 
SEAC remains controversial., ${ }^{2,5)}$

Here we report our experience with 2 SEAC cases and review the relevant current literature.

\section{Case Report}

\section{Case 1}

A 72-year-old woman presented with low back pain for 10 years and bilateral leg radiating pain for 4 months. She had no motor weakness and no pathological reflexes. She was first diagnosed with a SEAC 10 years ago at the local medical center and treated with medication.

We performed a radiological examination of the patient, including myelography, computed tomography (CT), and magnetic resonance imaging (MRI).

Preoperative myelography showed multi-level spinal canal stenosis and distortion from T12 to L5 on the spinal level. No connection site was found between the cyst and the thecal sac (Figure 1A). A spinal MRI revealed an elongated well-defined mass-like lesion with a high T2 signal and a low T1 signal at T12 to L4 on the spinal level causing spinal stenosis (Figure 1B and C). We suspected that the dural defect was at L1 on the vertebral level because the presence of a CSF flow artifact.

We opened the L2 lamina site and the cyst was identified. There was no dural defect on the L2 level. We extended the incision line to the T11 level, and a T12 L1 hemi-laminectomy was performed. Eventually, we found a dural defect on the right side at the L1 vertebral level. We removed both the $\mathrm{L} 1$ spinous process and the L1 lamina to expose the defect site (Figure 2A). We performed a cyst penetration and a primary dural repair with the cyst wall
(Figure 2B).

The postoperative histologic findings were compatible with the presence of an arachnoid cyst with dystrophic calcification.

The patient's symptoms gradually subsided, and followup MRI scans taken 4 months after the surgery showed a
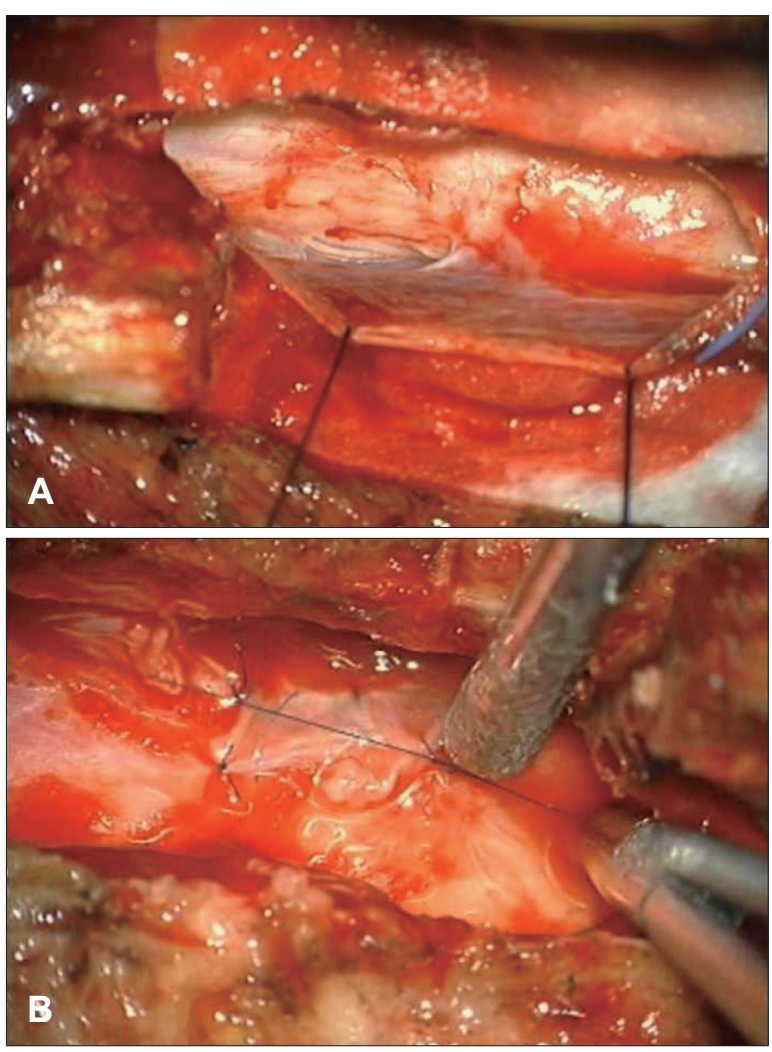

FIGURE 2. Intraoperative images of case 1. (A) L1 total laminectomy was performed to exposure dura defect. (B) Repair the defect site with the cyst wall.
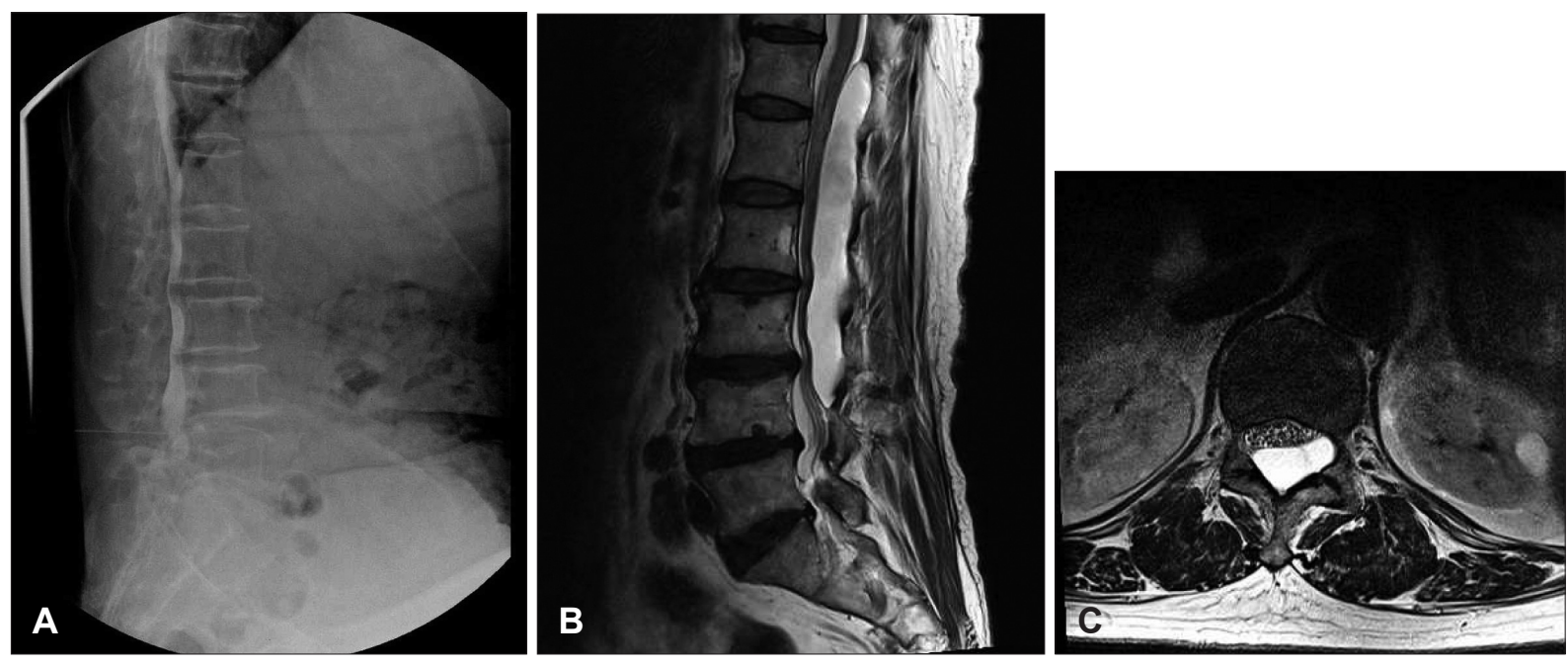

FIGURE 1. Preoperative images case 1. (A) Multi-level distortion of spinal canal and filling defect from T12 to L5 on myelogram. (B, C) Elongated cystic lesion compressing spinal thecal sac at T12 to L4. 
complete disappearance of the cyst with no evidence of cord compression due to a residual cyst (Figure 3).

\section{Case 2}

A 33-year-old woman presented with low back pain and right leg radiating pain for 7 months. She has no motor weakness and no pathological reflexes, but there was generalized paresthesia of the right leg. A SEAC was diagnosed using MRI at local medical center. The local hospital transferred the patient to our care for surgical treatment of the SEAC.

To prepare the operation, we performed an additional radiological examination, including a thoracolumbar spine simple X-ray and CT thoracic myelography scan.

Myelography revealed an arachnoid cyst at the posterior epidural space of T12 to L2, and indicated that communication of the cyst and the thecal sac occurred at the L1 level (Figure 4A). An MRI revealed an elongated well-defined mass-like lesion with a high $\mathrm{T} 2$ signal and a low $\mathrm{T} 1$ signal at T12 to L2 on the spinal level indicating thecal sac compression (Figure 4B and C).

First, we opened the T12 lamina site and performed a left partial hemi-laminectomy. The cyst wall was identified, and we identified a dural defect at the left L1 nerve root sleeve. However, we could not repair the defect due to spatial limitations. We had to remove T12 and L1 lamina to expose the defective site. We performed a cyst penetration and a primary dural repair with the cyst wall fragment (Figure 5).

Postoperative histologic findings were compatible the presence of an arachnoid cyst with fibrous cyst wall-like tissue.

Radiating leg pain and back pain in the patient were gradually improved, and a follow-up MRI taken 2 months after the surgery showed a complete disappearance of the cyst with no evidence of cord compression due to a residual cyst (Figure 6).

FIGURE 3. Postoperative images of case 1. (A, B) Magnetic resonance imaging taken 4 months after the surgery showed total removed state of cystic lesion.
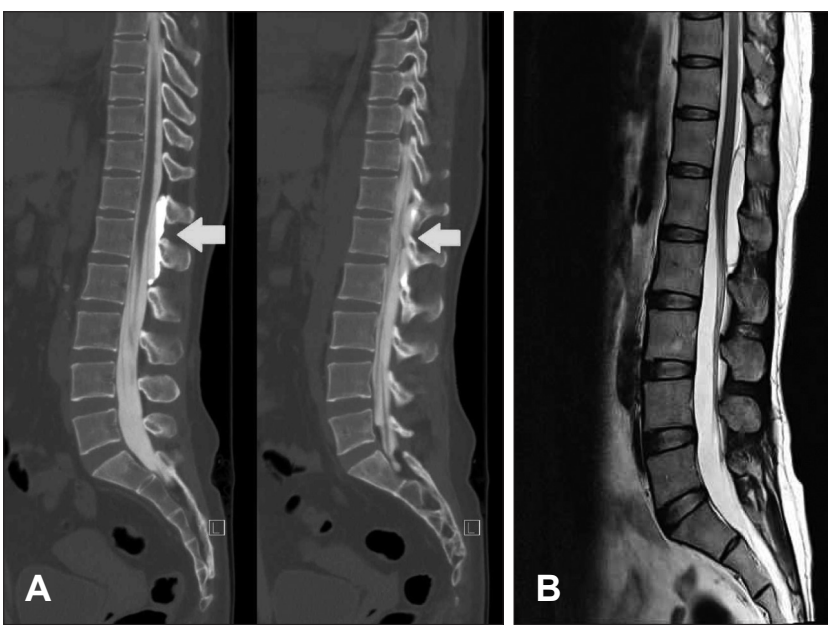

FIGURE 4. Preoperative images case 2. (A) Computed tomography myelography showed focal enhanced cystic mass that communicated with thecal sac at $L 1$ level. $(B, C)$ Cystic mass compressing dorsal side of thecal sac from T2 to $L 2$ level. 


\section{Discussion}

A SEAC is a rare spinal tumor that does not expand within the spinal canal. ${ }^{1-9)}$ A SEAC may develop as a cystic formation that is caused by a dural defect, and may be enlarged by the flow of CSF that originates from the intradural arach-
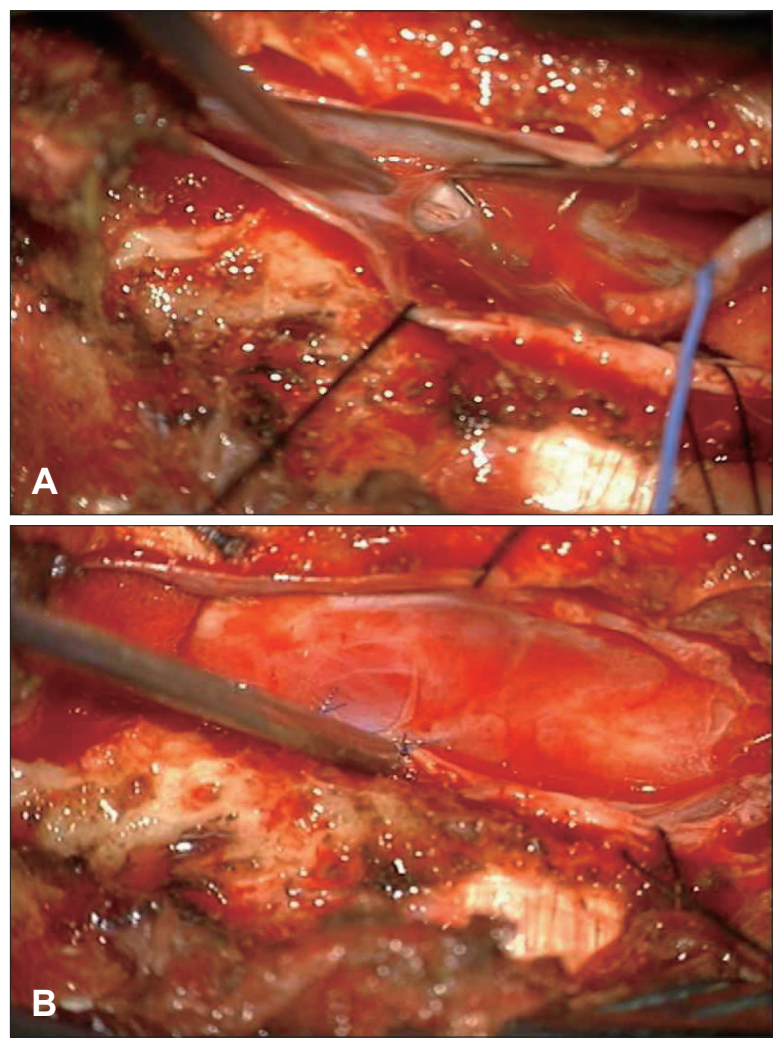

FIGURE 5. Intraoperative images of case 2. (A) Total laminectomy T12 and L1 level was performed to exposure the defective site. (B) Using cyst wall fragment, primary repair of defective site was performed. noid space. ${ }^{1,6)}$ A SEAC may grow extraspinally or intraspinally, and is also typically located on the posterolateral portion of the spinal canal, compressing the spinal cord toward the anterior side. ${ }^{1,6,8,9)}$ As enlargement continues, a SEAC can aggravate spinal cord compression or nerve root compression, which leads to patient symptoms such as pain or weakness. $^{1,6,8,9)}$

Although many have studied the etiology of theses cysts, such mechanisms remain uncertain. ${ }^{1-9)}$ For nearly all SEAC cases, communication between the subarachnoid spaces and cysts have been reported. ${ }^{1-3,5,8)}$ This communication can be congenital or acquired. A small dural tear that is induced by a congenital anomaly, trauma, arachnoiditis, or iatrogenic events allows for CSF flow to initiate herniation of the arachnoid membrane. The dural defect is usually found near the nerve root sleeve. ${ }^{1,2,6)}$ The most reliable theory of etiology of such tears suggests that stretching force between the movable thecal sac and the relatively fixed roots are a major factor. ${ }^{1)}$ The possibility of a dural tear increases if the patients has underlying structural problems (e.g., Marfan syndrome or dural ectasia) or thecal sac movements (trauma or CSF leakage). ${ }^{1,6,7)}$ Our current understanding is that this small dural tear appears to act as a ball-valve, and active CSF secretion from the residual arachnoid membrane plays a role in cyst expansion. ${ }^{1)}$ This cyst expansion can cause compressive myelopathy.

In our cases, neither patient had a history of trauma or arachnoiditis, or a previous spine surgery. The patient in case 1 had hypertension and hyperlipidemia, but no structural diseases. The patient in case 2 did not have any specific underlying diseases. The cysts observed in both cases showed a single small dural tear at the L1 right root level,

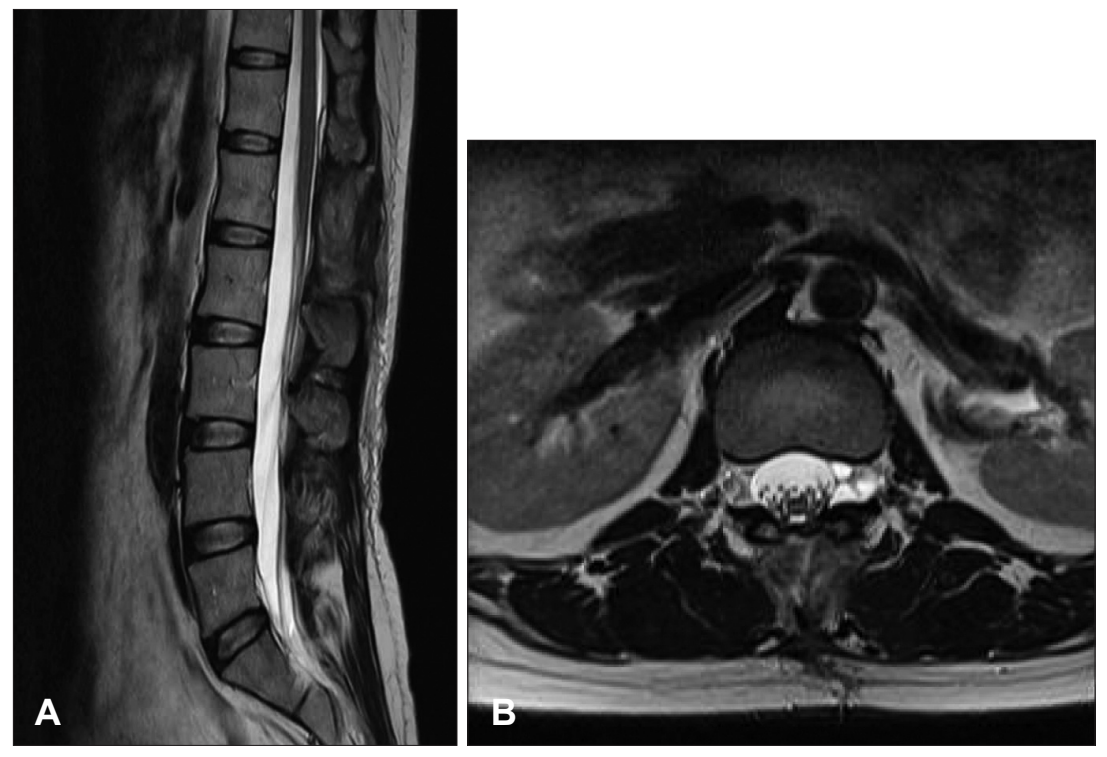

FIGURE 6. Postoperative images of case 2. (A, B) Magnetic resonance imaging taken 2 months after the surgery showed a complete disappearance of the cyst with no evidence of cord compression due to a residual cyst. 
and a trapped rootlet was found near the dural defect. We suggest that the CSF flow via the dural defect and the rootlet trapped in the defect likely acts as a one-way-valve mechanism, which has been described in several studies. The cysts in our cases are similar to a Type 2 meningeal cyst that was observed in another study. Histological investigation showed that the cyst wall in our cases only contains calcified tissue and layered collagenous fibers, but not the glands or secreting tissues.

Since the clinical symptoms of SEACs are similar to those for other degenerative spine diseases, SEACs are easy to misdiagnose. An MRI is the most useful tool to diagnose a SEAC. ${ }^{6)}$ Radiological studies report that a SEAC appears with a low signal intensity on a T1-weighted image (WI) and with a high signal intensity on a T2-WI, similar to cerebrospinal fluid. ${ }^{1,2,6,8)}$ A CT myelography is also a useful diagnostic tool because it can more reliably detect the anatomical location of the cyst, and measures the severity of compression of the spinal cord and nerve roots. ${ }^{1)}$ In addition, some reports indicate that a myelogram and CT myelography can help locate the dural defective site between the spinal subarachnoid space and the cyst cavity. ${ }^{1,9)}$

MRI methods that detect CSF flow can help to localize a defective site. A study reported that CSF flow MRI can identify the pulsating turbulent flow void of a defective site. ${ }^{1)}$ In our cases, preoperative CT myelography could not help to detect the dural defect site. However, we could predict where the location of the dural defect before surgery using a CSF flow MRI, and we could identify the exact location during surgery.

The standard treatment for SEACs remains uncertain.- ${ }^{1-3,5,6,8,9)}$ Some recommend non-surgical care for the treatment of SEACs and have reported good outcomes with this approach. ${ }^{1,6)}$ However, if the patient has a neurologic symptom that is caused by nerve compression due to a growing SEAC, then a surgical procedure would be needed. ${ }^{2,5)}$ There is a consensus among investigators to repair the dural defect in the treatment of a SEAC.$^{1-3,6,9)}$ However, there is still disagreement regarding the treatment of the cyst.

Some insist that the complete removal of the cyst is necessary to prevent cyst recurrence. ${ }^{5)}$ Other insist that a SEAC can be removed with only fenestration of the cystic wall. ${ }^{1}$ Yet another investigator implanted a shunt or aspirated the cyst. ${ }^{5)}$ Traditionally, a complete microsurgical resection of a SEAC with multilevel laminectomy and repair of dural defect has been promoted as the standard treatment of choice for a SEAC. ${ }^{1)}$ However, if the cyst is large, an extensive laminectomy would be necessary to remove the entire cyst wall for neural decompression. However, such a procedure can cause postoperative complications such as bleeding and postoperative instability. ${ }^{1-3,5,6,8,9)}$ Selective laminectomy with closure of the dural defect site alone has been reported as effective for treating an SEAC. ${ }^{1,6)}$ In our cases, we performed selective laminectomy with interlaminar fenestration at the transdural communication site that was observed as a pulsating flow, voiding on a preoperative MRI.

With the repair of the dural defect site in our cases, the regression of the cyst was confirmed in the postoperative radiological finding, and the clinical symptoms were significantly improved. Our procedure of selective laminectomy with interlaminar fenestration has the advantage of being a limited laminectomy, which can prevent complications related to wide surgical operation. However, this approach requires localization of the dural defect beforehand. Because of the technical precision of current radiological diagnostic devices, we could accurately localize the communication site, and minimize the extent of laminectomy and risk of complication. Our approach of focal laminectomy for cyst fenestration and repair of the dural defect produced good clinical and radiological outcomes.

\section{Conclusion}

A SEAC, which is mostly found at the thoracic spine, is a rare disease. A SEAC can cause spinal cord or nerve root compression and may present with myelopathy.

A SEAC is caused by the herniation of the arachnoid membrane, which is due to a dural defect that can be generated by congenital anomaly, trauma, infection, or inflammation. The causes of such herniation remain unclear.

To treat an SEAC, diverse surgical techniques have been introduced. However, there is no consensus regarding the most reasonable treatment. Most investigators agree that the dural defect must be repaired, but total removal of the SEAC is controversial.

We penetrated the cyst and performed a primary repair of the dural defect for two SEAC cases.

Compared to the classical complete resection of the cyst wall with multilevel laminectomy, our approach of fenestration to treat a SEAC poses fewer complications.

- The authors have no financial conflicts of interest.

\section{REFERENCES}

1) Choi SW, Seong HY, Roh SW. Spinal extradural arachnoid cyst. J Korean Neurosurg Soc 54:355-358, 2013

2) Funao $H$, Nakamura M, Hosogane N, Watanabe $K$, Tsuji T, Ishii K, et al. Surgical treatment of spinal extradural arachnoid cysts in the thoracolumbar spine. Neurosurgery 71:278-284, 2012

3) Furtado SV, Thakar S, Murthy GK, Dadlani R, Hegde AS. Man- 
agement of complex giant spinal arachnoid cysts presenting with myelopathy. J Neurosurg Spine 15:107-112, 2011

4) Kanetaka M, Sugita S, Chikuda H, Takeshita K, Ono T, Oshima $\mathrm{Y}$, et al. Use of Doppler ultrasonography to detect an elusive communication of a spinal extradural arachnoid cyst. J Clin Neurosci 18:863-864, 2011

5) Lee CH, Hyun SJ, Kim KJ, Jahng TA, Kim HJ. What is a reasonable surgical procedure for spinal extradural arachnoid cysts: is cyst removal mandatory? Eight consecutive cases and a review of the literature. Acta Neurochir (Wien) 154:1219-1227, 2012

6) Netra R, Min L, Shao Hui M, Wang JC, Bin Y, Ming Z. Spinal ex- tradural meningeal cysts: an MRI evaluation of a case series and literature review. J Spinal Disord Tech 24:132-136, 2011

7) Ogura Y, Yabuki S, Iida A, Kou I, Nakajima M, Kano H, et al. FOXC2 mutations in familial and sporadic spinal extradural arachnoid cyst. PLoS One 8:e80548, 2013

8) Oh JK, Lee DY, Kim TY, Yi S, Ha Y, Kim KN, et al. Thoracolumbar extradural arachnoid cysts: a study of 14 consecutive cases. Acta Neurochir (Wien) 154:341-348, 2012

9) Panigrahi S, Mishra SS, Dhir MK, Parida DK. Giant thoracolumbar extradural arachnoid cyst: an uncommon cause of spine compression. Neurol India 60:540-542, 2012 